\title{
Altered Microstructure in Temporal Lobe Epilepsy: A Diffusional Kurtosis Imaging Study
}

\author{
L. Bonilha, C.-Y. Lee, J.H. Jensen, A. Tabesh, M.V. Spampinato, J.C. Edwards, J. Breedlove, and J.A. Helpern
}

\begin{abstract}
BACKGROUND AND PURPOSE: Temporal lobe epilepsy is associated with regional abnormalities in tissue microstructure, as demonstrated by DTI. However, the full extent of these abnormalities has not yet been defined because DTI conveys only a fraction of the information potentially accessible with diffusion MR imaging. In this study, we assessed the added value of diffusional kurtosis imaging, an extension of DTI, to evaluate microstructural abnormalities in patients with temporal lobe epilepsy.
\end{abstract}

MATERIALS AND METHODS: Thirty-two patients with left temporal lobe epilepsy and 36 matched healthy subjects underwent diffusion MR imaging. To evaluate abnormalities in patients, we performed voxelwise analyses, assessing DTI-derived mean diffusivity, fractional anisotropy, and diffusional kurtosis imaging-derived mean diffusional kurtosis, as well as diffusional kurtosis imaging and DTI-derived axial and radial components, comparing patients with controls.

RESULTS: We replicated findings from previous studies demonstrating a reduction in fractional anisotropy and an increase in mean diffusivity preferentially affecting, but not restricted to, the temporal lobe ipsilateral to seizure onset. We also noted a pronounced pattern of diffusional kurtosis imaging abnormalities in gray and white matter tissues, often extending into regions that were not detected as abnormal by DTI measures.

CONCLUSIONS: Diffusional kurtosis is a sensitive and complementary measure of microstructural compromise in patients with temporal lobe epilepsy. It provides additional information regarding the anatomic distribution and degree of damage in this condition. Diffusional kurtosis imaging may be used as a biomarker for disease severity, clinical phenotypes, and treatment monitoring in epilepsy.

ABBREVIATIONS: $\mathrm{DKI}=$ diffusional kurtosis imaging; $\mathrm{FA}=$ fractional anisotropy; $\mathrm{MD}=$ mean diffusivity; $\mathrm{TLE}=$ temporal lobe epilepsy

$\mathbf{T}$ he most common histologic finding in patients with medial temporal lobe epilepsy (TLE) is hippocampal sclerosis, which is defined as neuronal loss and gliosis involving the hippocampus. ${ }^{1-3}$ Routine clinical MR imaging of patients with TLE can demonstrate signs associated with hippocampal sclerosis. ${ }^{4,5}$ Recent observations suggest that hippocampal abnormalities are not the only structural injury in TLE. ${ }^{6-9}$ Imaging studies

Received July 12, 2014; accepted after revision October 19.

From the Departments of Neurology and Neurosurgery (L.B., J.C.E.) and Radiology and Radiological Science (C.-Y.L., J.H.J., A.T., M.V.S., J.A.H.), Comprehensive Epilepsy Center (L.B., J.C.E., J.B.), and Center for Biomedical Imaging (L.B., C.-Y.L., J.H.J., A.T., M.V.S.), Medical University of South Carolina, Charleston, South Carolina.

This study was supported by the American Society of Neuroradiology (grant No. 89779-01; Principal Investigator, A. Tabesh).

Please address correspondence to Leonardo Bonilha, MD, PhD, Department of Neurology and Neurosurgery, Medical University of South Carolina, 96 Jonathan Lucas St, 3rd floor CSB, Charleston, SC 29425; e-mail: bonilha@musc.edu

三 Indicates article with supplemental on-line table.

http://dx.doi.org/10.3174/ajnr.A4185 using whole-brain quantitative morphometry have repeatedly demonstrated that TLE is associated with extrahippocampal abnormalities ${ }^{6,8-13}$ involving perihippocampal and perilimbic structures.

However, the full extent of structural abnormalities in TLE is still unclear. Therefore, the lack of a sensitive and specific marker of extrahippocampal damage may prevent the assessment of its clinical relevance. Diffusion MR imaging techniques aimed at quantifying tissue microstructure may offer more sensitive tools for determining the extent of brain pathology in TLE. A promising new method called diffusional kurtosis imaging (DKI) can provide information about cerebral microstructural abnormalities beyond that provided by conventional diffusion tensor imaging. ${ }^{14,15}$ In fact, by providing a more comprehensive characterization of water diffusion properties, DKI may be more suitable for detecting subtle brain damage in TLE.

In this study, we investigated the anatomical pattern of microstructural abnormalities associated with TLE by contrasting the 
voxel-based analyses of microstructure maps derived from DKI and from well-established DTI.

\section{MATERIALS AND METHODS \\ Subjects}

Thirty-two consecutive patients with left TLE were recruited from the Comprehensive Epilepsy Center at the Medical University of South Carolina, where they were diagnosed on the basis of a comprehensive history and neurologic evaluation. The TLE diagnosis was defined in accordance with the criteria proposed by the International League Against Epilepsy, ${ }^{16}$ based on a history compatible with partial seizures with temporal onset, interictal epileptiform discharges on interictal electroencephalography, and hippocampal atrophy on visual inspection of MR imaging. Not all patients included in this study were surgical candidates; thus, ictal recording was not obtained in all patients. All patients exhibited a classic presentation for TLE, based on history, seizure semiology, epileptiform discharges on interictal electroencephalography, as well as routine MRI. Most patients, but not all, exhibited signs of hippocampal sclerosis on MR imaging, and a detailed description of the demographic and clinical information for patients included in this study is provided in the On-line Table.

The mean age of patients was $44.8 \pm 16.7$ years; 22 patients were women. We also studied a control group of healthy individuals recruited from the local community who had no history of neurologic problems and no risk factors for epilepsy (mean age, $40.4 \pm 11.6$ years; 24 women). Patients and controls were similar in sex $(P=.21)$ and age $(P=.92)$ distributions.

\section{Image Acquisition}

All patients and controls underwent the same imaging protocol performed on a 3T Magnetom Verio MR imaging scanner (Siemens, Erlangen, Germany) equipped with a 12-channel head coil. Diffusion-weighted images were obtained by using a twice-refocused echo-planar sequence with 3 diffusion weightings ( $b=0$, 1000 , and $2000 \mathrm{~s} / \mathrm{mm}^{2}$ ) along 30 diffusion-encoding directions with $\mathrm{NEX}=1(\mathrm{NEX}=10$ for $b=0)$. Other imaging parameters were TR $=8500 \mathrm{~ms}, \mathrm{TE}=98 \mathrm{~ms}$, FOV $=222 \times 222 \mathrm{~mm}^{2}$, matrix size $=74 \times 74$, parallel imaging factor $=2$, no partial Fourier encoding, section thickness $=3 \mathrm{~mm}$, and 40 axial sections. Acquisition time was 9 minutes and 12 seconds. Structural images were obtained by using a magnetization-prepared rapid acquisition of gradient echo sequence with $\mathrm{TR}=2250 \mathrm{~ms}$, TE $=4.18 \mathrm{~ms}$, $\mathrm{TI}=900 \mathrm{~ms}, \mathrm{FOV}=256 \times 256 \mathrm{~mm}^{2}$, matrix size $=256 \times 256$, section thickness $=1 \mathrm{~mm}$, and 176 sagittal sections.

\section{Image Processing}

Tissue Volume Maps. T1-weighted images were submitted to gray and white matter tissue segmentation using the VBM toolbox (http://dbm.neuro.uni-jena.de/vbm/) for SPM8 software (http:// www.fil.ion.ucl.ac.uk/spm/software/spm8). Image normalization and segmentation were performed iteratively by using the symmetric tissue probability map of VBM, very light regularization, and affine regularization to the ICBM space template (European Brains; http://bmap.ucla.edu/portfolio/atlases/ICBM_Template/).Spatial normalization was performed with affine and nonlinear transformations yielding modulated normalized gray and white matter probability maps. All maps were transformed into standard space, and their averages (gray and white matter maps separately) were used to provide tissue inclusion masks for statistical analyses.

Microstructure Maps. DKI postprocessing was performed by using the in-house software Diffusional Kurtosis Estimator (http:// www.nitrc.org/projects/dke), ${ }^{17}$ which performed the following processing steps to generate diffusivity and kurtosis maps: first, motion correction through a 6-parameter rigid-body transformation to spatially align all DWIs. Second, at each voxel, the diffusion and diffusional kurtosis tensors were jointly fitted to the DWIs for $b=0,1000$, and $2000 \mathrm{~s} / \mathrm{mm}^{2}$ for that voxel, and the mean kurtosis was calculated from the tensors. The diffusion tensor was fitted to the DWIs for $b=0$ and $1000 \mathrm{~s} / \mathrm{mm}^{2}$, and mean diffusivity and (MD) fractional anisotropy (FA) were calculated from the diffusion tensor. Axial and radial diffusion and kurtosis maps were also generated.

FA maps were nonlinearly normalized to a common space by using the fMRI of the Brain Software Library, Version 4.1.7 (http://www.fmrib.ox.ac.uk/fsl) using a symmetric FA template, and the resulting transformation was applied to normalize the other maps. The spatially normalized images were then submitted to spatial smoothing with an 8-mm Gaussian filter.

Statistical Analyses. We performed whole-brain voxelwise analyses, comparing patients with TLE and controls regarding MD, FA, mean kurtosis, axial diffusion, radial diffusion, axial kurtosis, and radial kurtosis maps. We performed comparisons using voxelwise $t$ tests. All results were corrected for multiple comparisons using a false discovery rate threshold of $q<0.01 .^{18}$

\section{RESULTS \\ Gray Matter}

Patients with TLE exhibited multiple cortical areas with significant increase in diffusion parameters, along with a concurrent decrease in kurtosis parameters.

Specifically, an increase in MD was observed within the orbitofrontal cortices, frontal poles, medial left temporal lobe region, and midposterior cingulate. No significant changes were observed with axial diffusion, but radial diffusion was increased in patients in the orbitofrontal regions, frontal poles, medial left temporal regions, left lateral temporal regions, and midposterior cingulate cortex.

In contrast, at the same statistical threshold level, a more diffuse pattern of significant reductions in mean kurtosis was observed, encompassing the temporopolar cortices, temporal and frontal opercula, medial temporal lobe regions (more pronounced on the left side), left lateral temporal region, prefrontal cortices and temporal poles, and cingulate and parietal regions. Overall, a higher statistical difference was noted on the left side.

Reductions in axial kurtosis were noted particularly over the frontal polar regions, left temporal pole, left lateral temporal cortex, and left precentral areas. Reductions in radial kurtosis were diffusely distributed across the entire brain but were particularly intense over the left medial temporal cortex, left temporal polar cortex, left insula, left fusiform gyrus, cingulate cortex, and left precentral regions.

These results are shown in Fig 1. 


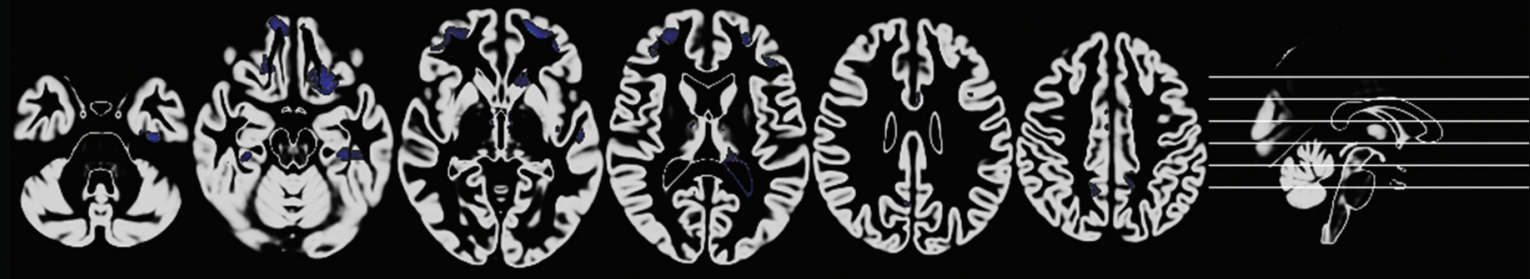

$\mathrm{D} \perp$

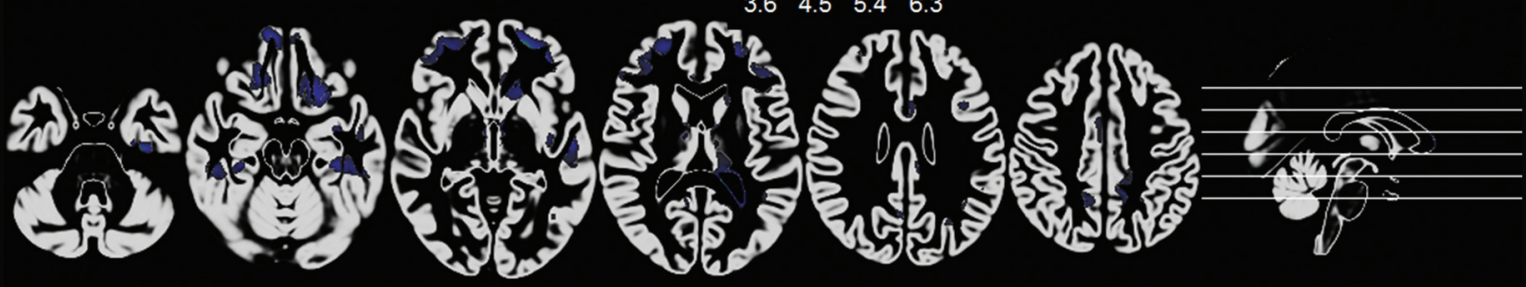

MK

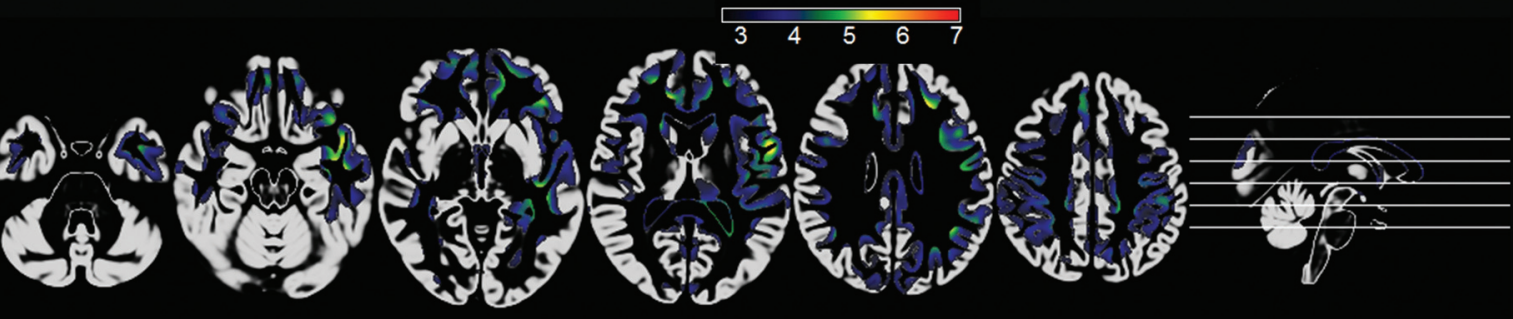

a
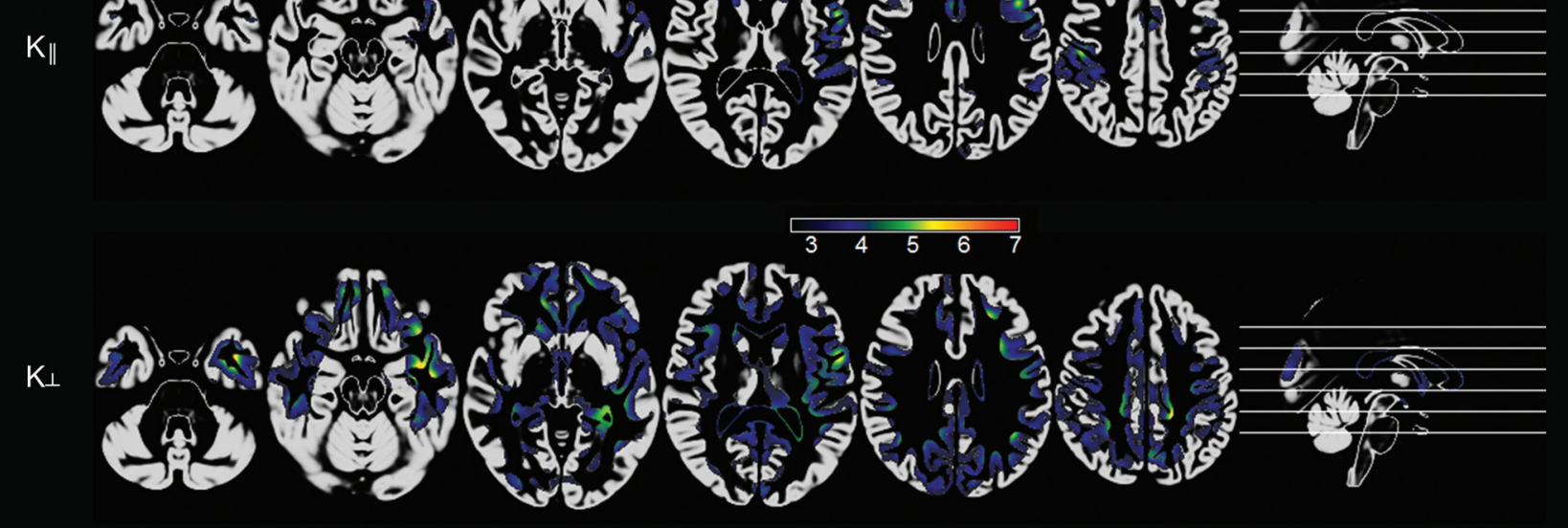

FIG 1. Voxelwise maps of cortical abnormalities in patients with TLE compared with controls. Statistical maps are overlaid on an average of probabilistic gray matter maps from all subjects. The scale bars represent absolute values of $t$ scores. The first row demonstrates areas with an increase in MD in patients. The second row demonstrates areas of increased radial diffusion in patients. The third, fourth, and fifth rows demonstrate areas of reduced mean kurtosis and axial and radial kurtosis, respectively, in patients. All results were corrected for multiple comparisons by using a false discovery rate threshold of $q<0.01$.

There were no cortical areas of reduced diffusion or increased kurtosis measures in patients.

\section{White Matter}

Patients with TLE demonstrated a significant increase in MD within the white matter in the frontal, left medial temporal, perithalamic, left occipitotemporal, and medial frontoparietal regions. There was also an increase in MD within the splenium of the corpus callosum. These abnormalities were also noted in areas of radial diffusion decrement, while axial diffusion did not demonstrate significant abnormalities between groups.

FA decrement was noted in a widespread pattern involving the medial temporal, perithalamic, orbitofrontal, left temporopolar, left occipitotemporal, and medial frontoparietal white matter regions. There was also a notable decrement in FA within the splenium of the corpus callosum, left side of the genu of the corpus callosum, and left uncinate fasciculus.

At the same statistical threshold, there was a remarkable ana- 


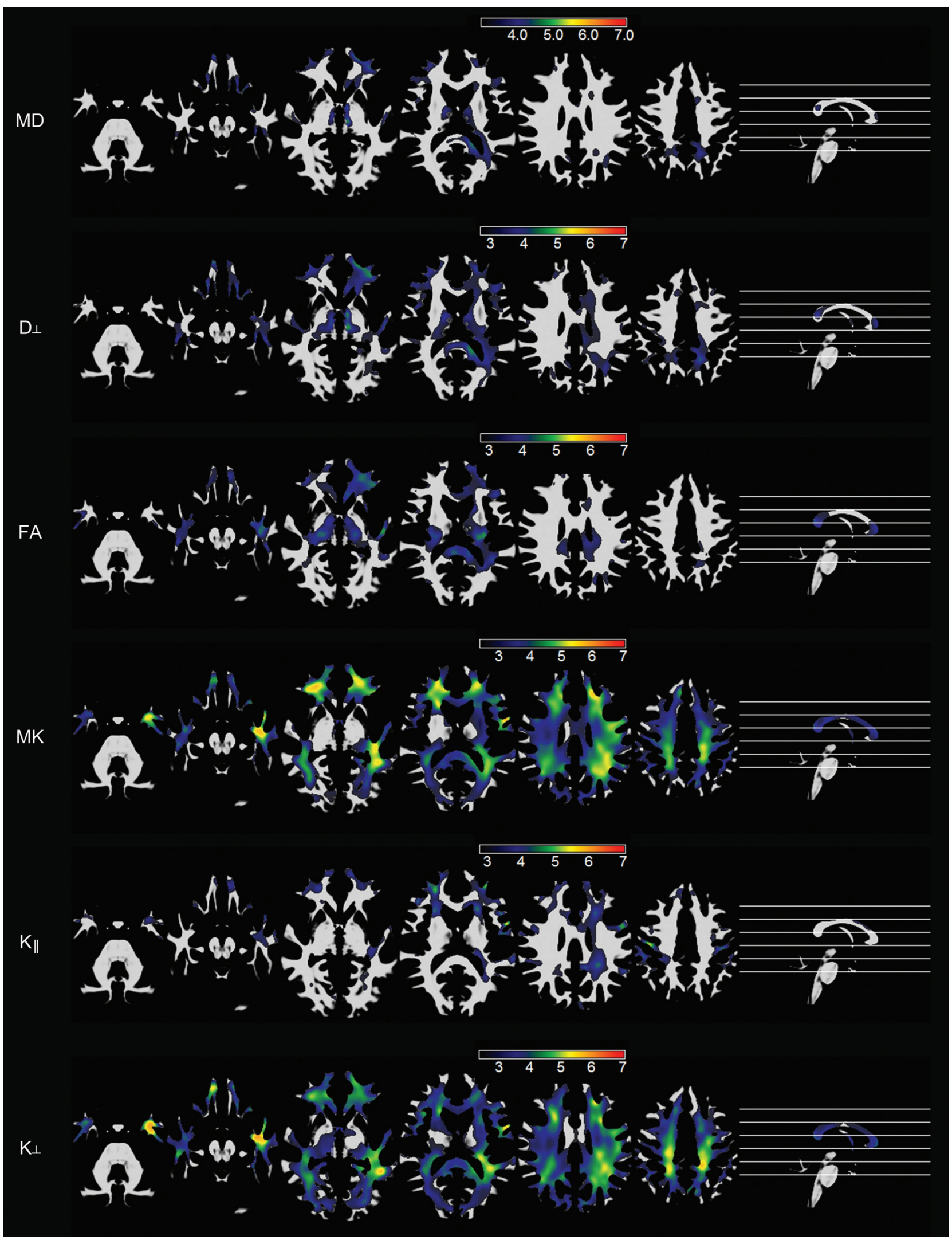

FIG 2. Voxelwise maps of white matter abnormalities in patients with TLE compared with controls. Statistical maps are overlaid on an average of probabilistic white matter maps from all subjects. The scale bars represent absolute values of $t$ scores. The first and second rows demonstrate areas with an increase in MD and radial diffusion, respectively, in patients. The third row demonstrates areas with reduced FA in patients, while the fourth, fifth, and sixth rows demonstrate areas of reduced mean kurtosis, axial kurtosis, and radial kurtosis in patients, respectively. All results were corrected for multiple comparisons by using a false discovery rate threshold of $q<0.01$. 
tomical pattern of mean kurtosis reduction, which involved most of the brain white matter and was most pronounced over the left temporal, bilateral orbitofrontal, and left frontoparietal regions. These abnormalities were similar to the pattern noted with radial kurtosis decrement in patients.

These results are shown in Fig 2.

There were no areas of MD decrement, FA increase, or kurtosis increase in patients.

\section{DISCUSSION}

In this study, we investigated microstructural gray and white matter abnormalities in patients with TLE using a novel diffusion MR imaging technique called DKI. DKI is aimed at detecting changes in tissue microstructure, including those arising from non-Gaussian properties of water diffusion, thus providing a potentially more sensitive and specific tool for the evaluation of pathologic changes in TLE. ${ }^{14,15}$ We contrasted the DKI-derived microstructural measures with the better established DTI-derived measures.

We replicated findings from several previous studies using DTI to assess microstructural abnormalities in TLE. Specifically, we demonstrated areas of increased MD and reduced FA in patients with TLE, which were preferentially located within, but not restricted to, the temporal lobe ipsilateral to the side of seizure onset. $^{19-29}$

We observed that DKI is capable of detecting a broader anatomic pattern of microstructural abnormalities in patients with TLE, compared with conventional measures. Moreover, these results demonstrate a distribution of network abnormalities that is in direct concordance with the hypothesized network pathology related to TLE. ${ }^{13,30}$ The presence of abnormalities demonstrated by DKI is more contiguous and anatomically plausible compared with conventional methodologies, and it is consistent with the theory of limbic and perilimbic dysfunction in TLE. ${ }^{31,32}$

In this study, we also corroborated the findings from Gao et $\mathrm{al},{ }^{33}$ suggesting that DKI is more sensitive, compared with DTI, to detect microstructural abnormalities in the patients with TLE. Gao et al studied a population of children with TLE; nonetheless, their results are fairly equivalent to our observations from a population of adult subjects with TLE.

\section{DKI and the Pathophysiology of TLE}

The hypothesis that TLE is a disease that affects more than just the hippocampus has been corroborated by several imaging studies demonstrating abnormalities in TLE involving limbic structures. ${ }^{6,8-13}$ However, there is considerable variability across studies regarding the extent and degree of extrahippocampal damage in TLE. These variations may be directly related to the sensitivity of the methods, as further highlighted by the observation that conventional methods are not sensitive to most extrahippocampal histologic changes. ${ }^{34,35}$ This variability in findings precludes a better understanding of the pathophysiology of TLE.

Our results suggest that TLE is associated with extensive microstructural abnormalities, encompassing a contiguous and extensive network of extrahippocampal and extratemporal regions.

Our results were obtained from a population of consecutive patients with TLE, most of whom demonstrated signs of hippocampal sclerosis on MR imaging (On-line Table). Therefore, it is possible that the temporal and extratemporal microstructural abnormalities demonstrated by DKI are correlated with hippocampal cell loss. Future studies evaluating phenotypical differences in the distribution of DKI abnormalities may provide further insight into this subject.

Moreover, the patient population evaluated in this study was not composed exclusively of surgical candidates (ie, it included patients with relatively well-controlled epilepsy). Most interesting, our strong statistical results suggest that microstructural abnormalities were widespread across all subjects. While these findings support DKI as exquisitely sensitive to abnormalities associated with TLE, it is still unclear whether DKI can provide further information regarding classification into subgroups, particularly as it relates to pharmacologic or surgical treatment outcomes. This study provides initial evidence of DKI as a biomarker of TLE, and we believe that future studies could address the impact of DKI as a clinical biomarker.

\section{DKI as a Biomarker}

The results of this study suggest that DKI may provide a sensitive and specific biomarker of TLE. Specifically, it may be a powerful tool for determining the degree of abnormality in cortical regions, which may be related to the clinical or neuropsychological profiles, and for quantifying the burden of overall network abnormalities on clinical progression. Specifically, extrahippocampal microstructural tissue abnormalities may be related to epileptogenesis in some individuals with TLE. ${ }^{31,36,37}$ These microstructural changes may represent a complex interaction among multiple pathologic mechanisms such as cell loss, inflammation, and axonal and dendritic reorganization, which are known to occur in epilepsy. ${ }^{38}$

\section{Clinical Feasibility of DKI}

DKI provides a convenient platform for the evaluation of microstructural abnormalities related to TLE and is one form of advanced diffusion MR imaging. ${ }^{39}$ However, DKI may be advantageous to other methods due to its feasibility in routine clinical practice. Other modalities require significantly longer scan times and/or custom hardware. Conversely, DKI can be acquired with a high signal-to-noise ratio in approximately 6-10 minutes, depending on the scanner.

\section{Future Applications}

This study provides preliminary evidence in support of the utility of DKI for characterization of microstructural abnormalities in TLE. Our results suggest that DKI-defined microstructural abnormalities are pervasive in the hippocampal, temporal, and extratemporal regions. Future studies should further investigate the histologic correlates of these abnormalities and their relationship with the clinical and neuropsychological profiles in patients with medial temporal lobe epilepsy.

\section{CONCLUSIONS}

This study demonstrates the added value of kurtosis measures in the assessment of microstructural alterations in adult patients with TLE. The results of this study confirm our hypothesis that 
DKI may provide complementary information regarding the location and magnitude of structural abnormalities in TLE.

Disclosures: Leonardo Bonilha—RELATED: Grant: American Society of Neuroradiology (grant No. 89779-01, Principal Investigator, A. Tabesh).* Jens H. Jensen-OTHER RELATIONSHIPS: Siemens owns a royalty-free nonexclusive license for diffusional kurtosis imaging with the pending patent held by New York University. I am one of the inventors. Maria V. Spampinato-UNRELATED: Grants/Grants Pending: Bracco, * Comments: clinical trial on 2 FDA-approved contrast agents. Ali Tabesh-RELATED: Grant: American Society of Neuroradiology, ${ }^{*}$ Comments: 2013 Research Scientist Award. *Money paid to the institution.

\section{REFERENCES}

1. Sendrowski K, Sobaniec W. Hippocampus, hippocampal sclerosis and epilepsy. Pharmacol Rep 2013;65:555-65

2. Blümcke I, Coras R, Miyata H, et al. Defining clinico-neuropathological subtypes of mesial temporal lobe epilepsy with hippocampal sclerosis. Brain Pathol 2012;22:402-11

3. Babb TL, Brown WJ. Pathological findings in epilepsy. In: Engel JJ, ed. Surgical Treatment of the Epilepsies. New York: Raven; 1987: 511-40

4. Cendes F, Andermann F, Gloor P, et al. MRI volumetric measurement of amygdala and hippocampus in temporal lobe epilepsy. Neurology 1993;43:719-25

5. Lencz T, McCarthy G, Bronen RA, et al. Quantitative magnetic resonance imaging in temporal lobe epilepsy: relationship to neuropathology and neuropsychological function. Ann Neurol 1992;31: 629-37

6. Bernasconi N, Duchesne S, Janke A, et al. Whole-brain voxel-based statistical analysis of gray matter and white matter in temporal lobe epilepsy. Neuroimage 2004;23:717-23

7. Bonilha L, Kobayashi E, Rorden C, et al. Medial temporal lobe atrophy in patients with refractory temporal lobe epilepsy. J Neurol Neurosurg Psychiatry 2003;74:1627-30

8. Keller SS, Mackay CE, Barrick TR, et al. Voxel-based morphometric comparison of hippocampal and extrahippocampal abnormalities in patients with left and right hippocampal atrophy. Neuroimage 2002;16:23-31

9. McDonald CR, Hagler DJ Jr, Ahmadi ME, et al. Regional neocortical thinning in mesial temporal lobe epilepsy. Epilepsia 2008;49: 794-803

10. Bonilha L, Rorden C, Castellano G, et al. Voxel-based morphometry of the thalamus in patients with refractory medial temporal lobe epilepsy. Neuroimage 2005;25:1016-21

11. Bonilha L, Rorden C, Castellano G, et al. Voxel-based morphometry reveals gray matter network atrophy in refractory medial temporal lobe epilepsy. Arch Neurol 2004;61:1379-84

12. Bernasconi N, Bernasconi A, Caramanos Z, et al. Mesial temporal damage in temporal lobe epilepsy: a volumetric MRI study of the hippocampus, amygdala and parahippocampal region. Brain 2003; 126:462-69

13. Keller SS, Roberts N. Voxel-based morphometry of temporal lobe epilepsy: an introduction and review of the literature. Epilepsia 2008;49:741-57

14. Jensen JH, Helpern JA. MRI quantification of non-Gaussian water diffusion by kurtosis analysis. NMR Biomed 2010;23:698-710

15. Jensen JH, Helpern JA, Ramani A, et al. Diffusional kurtosis imaging: the quantification of non-Gaussian water diffusion by means of magnetic resonance imaging. Magn Reson Med 2005;53: $1432-40$

16. Proposal for revised classification of epilepsies and epileptic syndromes: Commission on Classification and Terminology of the International League Against Epilepsy. Epilepsia 1989;30:389-99

17. Tabesh A, Jensen JH, Ardekani BA, et al. Estimation of tensors and tensor-derived measures in diffusional kurtosis imaging. Magn Reson Med 2011;65:823-36

18. Genovese CR, Lazar NA, Nichols T. Thresholding of statistical maps in functional neuroimaging using the false discovery rate. Neuroimage 2002;15:870-78

19. Keller SS, Ahrens T, Mohammadi S, et al. Voxel-based statistical analysis of fractional anisotropy and mean diffusivity in patients with unilateral temporal lobe epilepsy of unknown cause. J Neuroimaging 2013;23:352-59

20. Keller SS, Ahrens T, Mohammadi S, et al. Microstructural and volumetric abnormalities of the putamen in juvenile myoclonic epilepsy. Epilepsia 2011;52:1715-24

21. Concha L, Beaulieu C, Collins DL, et al. White-matter diffusion abnormalities in temporal-lobe epilepsy with and without mesial temporal sclerosis. J Neurol Neurosurg Psychiatry 2009;80:312-19

22. Gong G, Concha L, Beaulieu C, et al. Thalamic diffusion and volumetry in temporal lobe epilepsy with and without mesial temporal sclerosis. Epilepsy Res 2008;80:184-93

23. Gross DW, Concha L, Beaulieu C. Extratemporal white matter abnormalities in mesial temporal lobe epilepsy demonstrated with diffusion tensor imaging. Epilepsia 2006;47:1360-63

24. Concha L, Beaulieu C, Gross DW. Bilateral limbic diffusion abnormalities in unilateral temporal lobe epilepsy. Ann Neurol 2005;57: $188-96$

25. Thivard L, Lehericy S, Krainik A, et al. Diffusion tensor imaging in medial temporal lobe epilepsy with hippocampal sclerosis. Neuroimage 2005;28:682-90

26. Kimiwada T, Juhasz C, Makki M, et al. Hippocampal and thalamic diffusion abnormalities in children with temporal lobe epilepsy. Epilepsia 2006;47:167-75

27. Focke NK, Yogarajah M, Bonelli SB, et al. Voxel-based diffusion tensor imaging in patients with mesial temporal lobe epilepsy and hippocampal sclerosis. Neuroimage 2008;40:728-37

28. Nilsson D, Go C, Rutka JT, et al. Bilateral diffusion tensor abnormalities of temporal lobe and cingulate gyrus white matter in children with temporal lobe epilepsy. Epilepsy Res 2008;81:128-35

29. Bonilha L, Edwards JC, Kinsman SL, et al. Extrahippocampal gray matter loss and hippocampal deafferentation in patients with temporal lobe epilepsy. Epilepsia 2010;51:519-28

30. Spencer SS. Neural networks in human epilepsy: evidence of and implications for treatment. Epilepsia 2002;43:219-27

31. Bonilha L, Martz GU, Glazier SS, et al. Subtypes of medial temporal lobe epilepsy: influence on temporal lobectomy outcomes? Epilepsia 2012;53:1-6

32. Richardson MP. Large scale brain models of epilepsy: dynamics meets connectomics. J Neurol Neurosurg Psychiatry 2012;83:1238-48

33. Gao Y, Zhang Y, Wong CS, et al. Diffusion abnormalities in temporal lobes of children with temporal lobe epilepsy: a preliminary diffusional kurtosis imaging study and comparison with diffusion tensor imaging. NMR Biomed 2012;25:1369-77

34. Eriksson SH, Free SL, Thom M, et al. Quantitative grey matter histological measures do not correlate with grey matter probability values from in vivo MRI in the temporal lobe. J Neurosci Methods 2009;181:111-18

35. Eriksson SH, Thom M, Symms MR, et al. Cortical neuronal loss and hippocampal sclerosis are not detected by voxel-based morphometry in individual epilepsy surgery patients. Hum Brain Mapp 2009;30:3351-60

36. Rorden C, Bonilha L, Nichols TE. Rank-order versus mean based statistics for neuroimaging. Neuroimage 2007;35:1531-37

37. Wennberg R, Arruda F, Quesney LF, et al. Preeminence of extrahippocampal structures in the generation of mesial temporal seizures: evidence from human depth electrode recordings. Epilepsia 2002; 43:716-26

38. Thom M, Eriksson S, Martinian L, et al. Temporal lobe sclerosis associated with hippocampal sclerosis in temporal lobe epilepsy: neuropathological features. J Neuropathol Exp Neurol 2009;68: $928-38$

39. Wedeen VJ, Hagmann P, Tseng WY, et al. Mapping complex tissue architecture with diffusion spectrum magnetic resonance imaging. Magn Reson Med 2005;54:1377-86 